\title{
$M^{2}$-Type sharp estimates and boundedness on a Morrey space for Toeplitz-type operators associated to singular integral operators satisfying a variant of Hörmander's condition
}

\section{Qiufen Feng*}

Correspondence:

fengqiufen@126.com

Changsha Commerce and Tourism

College, Yuhua District, White Road

No. 16, Changsha, Hunan, China

\section{Springer}

\begin{abstract}
In this paper, we prove the $\mathrm{M}^{2}$-type sharp maximal function estimates for the Toeplitz-type operators associated to certain singular integral operators satisfying a variant of Hörmander's condition. As an application, we obtain the weighted boundedness of the operators on the Lebesgue and Morrey spaces.

MSC: 42B20; 42B25
\end{abstract}

Keywords: Toeplitz-type operator; singular integral operator; sharp maximal function; BMO; Morrey space

\section{Introduction}

As the development of singular integral operators (see [1,2]), their commutators have been well studied. In $[3,4]$, the authors prove that the commutators generated by the singular integral operators and $B M O$ functions are bounded on $L^{p}\left(R^{n}\right)$ for $1<p<\infty$. Chanillo (see [5]) proves a similar result when singular integral operators are replaced by the fractional integral operators. In [6, 7], some Toeplitz-type operators related to the singular integral operators and strongly singular integral operators are introduced, and the boundedness for the operators generated by $B M O$ and Lipschitz functions is obtained. In [6], some singular integral operators satisfying a variant of Hörmander's condition are introduced, and the boundedness for the operators is obtained (see [6], [20]). In this paper, we prove the sharp maximal function inequalities for the Toeplitz-type operator related to some singular integral operators satisfying a variant of Hörmander's condition. As an application, we obtain the weighted boundedness of the Toeplitz-type operator on Lebesgue and Morrey spaces.

\section{Preliminaries}

First, let us introduce some notations. Throughout this paper, $Q$ will denote a cube of $R^{n}$ with sides parallel to the axes. For any locally integrable function $f$, the sharp maximal function of $f$ is defined by

$$
f^{\#}(x)=\sup _{Q \ni x} \frac{1}{|Q|} \int_{Q}\left|f(y)-f_{Q}\right| d y,
$$

@2013 Feng; licensee Springer. This is an Open Access article distributed under the terms of the Creative Commons Attribution License (http://creativecommons.org/licenses/by/2.0), which permits unrestricted use, distribution, and reproduction in any medium, provided the original work is properly cited. 
where, and in what follows, $f_{Q}=|Q|^{-1} \int_{Q} f(x) d x$. We say that $f$ belongs to $B M O\left(R^{n}\right)$ if $f^{\#}$ belongs to $L^{\infty}\left(R^{n}\right)$ and define $\|f\|_{B M O}=\left\|f^{\#}\right\|_{L^{\infty}}$. It has been known that (see [2])

$$
\left\|f-f_{2^{k} Q}\right\|_{B M O} \leq C k\|f\|_{B M O} .
$$

Let $M$ be the Hardy-Littlewood maximal operator defined by

$$
M(f)(x)=\sup _{Q \ni x} \frac{1}{|Q|} \int_{Q}|f(y)| d y .
$$

For $\eta>0$, let $M_{\eta}(f)=M\left(|f|^{\eta}\right)^{1 / \eta}$. For $k \in N$, we denote by $M^{k}$ the operator $M$ iterated $k$ times, i.e., $M^{1}(f)=M(f)$ and

$$
M^{k}(f)=M\left(M^{k-1}(f)\right) \quad \text { when } k \geq 2 .
$$

Let $\Phi$ be a Young function and $\tilde{\Phi}$ be the complementary associated to $\Phi$. We denote the $\Phi$-average by, for a function $f$,

$$
\|f\|_{\Phi, Q}=\inf \left\{\lambda>0: \frac{1}{|Q|} \int_{Q} \Phi\left(\frac{|f(y)|}{\lambda}\right) d y \leq 1\right\}
$$

and the maximal function associated to $\Phi$ by

$$
M_{\Phi}(f)(x)=\sup _{x \in Q}\|f\|_{\Phi, Q}
$$

The Young functions to be used in this paper are $\Phi(t)=t(1+\log t)$ and $\tilde{\Phi}(t)=\exp (t)$, the corresponding average and maximal functions denoted by $\|\cdot\|_{L(\log L), Q}, M_{L(\log L)}$ and $\|\cdot\|_{\exp L, Q}, M_{\exp L}$. Following [2], we know that the generalized Hölder inequality and the following inequalities hold:

$$
\begin{aligned}
& \frac{1}{|Q|} \int_{Q}|f(y) g(y)| d y \leq\|f\|_{\Phi, Q}\|g\|_{\tilde{\Phi}, Q}, \\
& \|f\|_{L(\log L), Q} \leq M_{L(\log L)}(f) \leq C M^{2}(f), \\
& \left\|f-f_{Q}\right\|_{\exp L, Q} \leq C\|f\|_{B M O}
\end{aligned}
$$

and

$$
\left\|f-f_{Q}\right\|_{\exp L, 2^{k} Q} \leq C k\|f\|_{B M O} .
$$

The $A_{p}$ weight is defined by (see [1])

$$
\begin{aligned}
A_{p} & =\left\{w \in L_{\mathrm{loc}}^{1}\left(R^{n}\right): \sup _{Q}\left(\frac{1}{|Q|} \int_{Q} w(x) d x\right)\left(\frac{1}{|Q|} \int_{Q} w(x)^{-1 /(p-1)} d x\right)^{p-1}<\infty\right\}, \\
& 1<p<\infty,
\end{aligned}
$$


and

$$
A_{1}=\left\{w \in L_{\mathrm{loc}}^{p}\left(R^{n}\right): M(w)(x) \leq C w(x), \text { a.e. }\right\}
$$

Given a weight function $w$, for $1 \leq p<\infty$, the weighted Lebesgue space $L^{p}(w)$ is the space of functions $f$ such that

$$
\|f\|_{L^{p}(w)}=\left(\int_{R^{n}}|f(x)|^{p} w(x) d x\right)^{1 / p}<\infty .
$$

Definition 1 Let $\Phi=\left\{\phi_{1}, \ldots, \phi_{l}\right\}$ be a finite family of bounded functions in $R^{n}$. For any locally integrable function $f$, the $\Phi$ sharp maximal function of $f$ is defined by

$$
M_{\Phi}^{\#}(f)(x)=\sup _{Q \ni x} \inf _{\left\{c_{1}, \ldots, c_{l}\right\}} \frac{1}{|Q|} \int_{Q}\left|f(y)-\sum_{i=1}^{l} c_{i} \phi_{i}\left(x_{Q}-y\right)\right| d y,
$$

where the infimum is taken over all $m$-tuples $\left\{c_{1}, \ldots, c_{l}\right\}$ of complex numbers and $x_{Q}$ is the center of $Q$. For $\eta>0$, let

$$
M_{\Phi, \eta}^{\#}(f)(x)=\sup _{Q \ni x} \inf _{\left\{c_{1}, \ldots, c_{l}\right\}}\left(\frac{1}{|Q|} \int_{Q \mid}\left|f(y)-\sum_{i=1}^{l} c_{j} \phi_{i}\left(x_{Q}-y\right)\right|^{\eta} d y\right)^{1 / \eta} .
$$

Remark We note that $M_{\Phi}^{\#} \approx f^{\#}$ if $l=1$ and $\phi_{1}=1$.

Definition 2 Given a positive and locally integrable function $f$ in $R^{n}$, we say that $f$ satisfies the reverse Hölder condition (write this as $f \in R H_{\infty}\left(R^{n}\right)$ ) if for any cube $Q$ centered at the origin, we have

$$
0<\sup _{x \in Q} f(x) \leq C \frac{1}{|Q|} \int_{Q} f(y) d y
$$

Definition 3 Let $\varphi$ be a positive, increasing function on $R^{+}$, and there exists a constant $D>0$ such that

$$
\varphi(2 t) \leq D \varphi(t) \quad \text { for } t \geq 0
$$

Let $w$ be a weight function and $f$ be a locally integrable function on $R^{n}$. Set, for $1 \leq p<\infty$,

$$
\|f\|_{L^{p, \varphi}(w)}=\sup _{x \in R^{n}, d>0}\left(\frac{1}{\varphi(d)} \int_{Q(x, d)}|f(y)|^{p} w(y) d y\right)^{1 / p}
$$

where $Q(x, d)=\left\{y \in R^{n}:|x-y|<d\right\}$. The generalized Morrey space is defined by

$$
L^{p, \varphi}\left(R^{n}, w\right)=\left\{f \in L_{\mathrm{loc}}^{1}\left(R^{n}\right):\|f\|_{L^{p, \varphi}(w)}<\infty\right\} .
$$

If $\varphi(d)=d^{\eta}, \eta>0$, then $L^{p, \varphi}\left(R^{n}, w\right)=L^{p, \eta}\left(R^{n}, w\right)$, which is the classical weighted Morrey spaces (see [8, 9]). If $\varphi(d)=1$, then $L^{p, \varphi}\left(R^{n}, w\right)=L^{p}\left(R^{n}, w\right)$, which is the weighted Lebesgue spaces (see [6]). 
As the Morrey space may be considered as an extension of the Lebesgue space, it is natural and important to study the boundedness of the operator on the Morrey spaces (see [5, 8-11]).

In this paper, we study some singular integral operators as follows (see [12]).

Definition 4 Let $K \in L^{2}\left(R^{n}\right)$ and satisfy

$$
\begin{aligned}
& \|K\|_{L^{\infty}} \leq C, \\
& |K(x)| \leq C|x|^{-n},
\end{aligned}
$$

there exist functions $B_{1}, \ldots, B_{l} \in L_{\text {loc }}^{1}\left(R^{n}-\{0\}\right)$ and $\Phi=\left\{\phi_{1}, \ldots, \phi_{l}\right\} \subset L^{\infty}\left(R^{n}\right)$ such that $\left|\operatorname{det}\left[\phi_{j}\left(y_{i}\right)\right]\right|^{2} \in R H_{\infty}\left(R^{n l}\right)$, and for a fixed $\delta>0$ and any $|x|>2|y|>0$,

$$
\left|K(x-y)-\sum_{i=1}^{l} B_{i}(x) \phi_{i}(y)\right| \leq C \frac{|y|^{\delta}}{|x-y|^{n+\delta}} .
$$

For $f \in C_{0}^{\infty}$, we define the singular integral operator related to the kernel $K$ by

$$
T(f)(x)=\int_{R^{n}} K(x-y) f(y) d y .
$$

Moreover, let $b$ be a locally integrable function on $R^{n}$. The Toeplitz-type operator related to $T$ is defined by

$$
T^{b}=\sum_{j=1}^{m} T^{j, 1} M_{b} T^{j, 2},
$$

where $T^{j, 1}$ are $T$ or $\pm I$ (the identity operator), $T^{j, 2}$ are the bounded linear operators on $L^{p}(w)$ for $1<p<\infty$ and $w \in A_{1}, j=1, \ldots, m, M_{b}(f)=b f$.

Remark Note that the classical Calderón-Zygmund singular integral operator satisfies Definition 4 (see [13], [19]). Also note that the commutator $[b, T](f)=b T(f)-T(b f)$ is a particular operator of the Toeplitz-type operators $T^{b}$. The Toeplitz-type operators $T^{b}$ are the non-trivial generalizations of the commutator. It is well known that commutators are of great interest in harmonic analysis and have been widely studied by many authors $($ see $[12,14])$. The main purpose of this paper is to prove the sharp maximal inequalities for the Toeplitz-type operator $T^{b}$. As the application, we obtain the weighted $L^{p}$-norm inequality and Morrey space boundedness for the Toeplitz-type operators $T^{b}$.

\section{Theorems and lemmas}

We shall prove the following theorems.

Theorem 1 Let $T$ be the singular integral operator as Definition 4, $0<r<1$ and $b \in$ $B M O\left(R^{n}\right)$. If $T^{1}(g)=0$ for any $g \in L^{u}\left(R^{n}\right)(1<u<\infty)$, then there exists a constant $C>0$ such that, for any $f \in C_{0}^{\infty}\left(R^{n}\right)$ and $\tilde{x} \in R^{n}$,

$$
M_{\Phi, r}^{\#}\left(T^{b}(f)\right)(\tilde{x}) \leq C\|b\|_{B M O} \sum_{j=1}^{m} M^{2}\left(T^{j, 2}(f)\right)(\tilde{x}) .
$$


Theorem 2 Let $T$ be the singular integral operator as Definition $4,1<p<\infty, w \in A_{1}$ and $b \in B M O\left(R^{n}\right)$. If $T^{1}(g)=0$ for any $g \in L^{u}\left(R^{n}\right)(1<u<\infty)$, then $T^{b}$ is bounded on $L^{p}(w)$.

Theorem 3 Let $T$ be the singular integral operator as Definition $4,0<D<2^{n}, 1<p<\infty$, $w \in A_{1}$ and $b \in B M O\left(R^{n}\right)$. If $T^{1}(g)=0$ for any $g \in L^{u}\left(R^{n}\right)(1<u<\infty)$, then $T^{b}$ is bounded on $L^{p, \varphi}\left(R^{n}, w\right)$.

To prove the theorems, we need the following lemmas.

Lemma 1 ([1, p.485]) Let $0<p<q<\infty$ and for any function $f \geq 0$. We define that for $1 / r=1 / p-1 / q$,

$$
\begin{aligned}
& \|f\|_{W L^{q}}=\sup _{\lambda>0} \lambda\left|\left\{x \in R^{n}: f(x)>\lambda\right\}\right|^{1 / q}, \\
& N_{p, q}(f)=\sup _{E}\left\|f \chi_{E}\right\|_{L^{p}} /\left\|\chi_{E}\right\|_{L^{r}},
\end{aligned}
$$

where the sup is taken for all measurable sets $E$ with $0<|E|<\infty$. Then

$$
\|f\|_{W L^{q}} \leq N_{p, q}(f) \leq(q /(q-p))^{1 / p}\|f\|_{W L^{q}} .
$$

Lemma 2 (see [2]) We have

$$
\frac{1}{|Q|} \int_{Q}|f(x) g(x)| d x \leq\|f\|_{\exp L, Q}\|g\|_{L(\log L), Q}
$$

Lemma 3 (see [15]) Let $T$ be the singular integral operator as Definition 4. Then $T$ is bounded on $L^{p}(w)$ for $1<p<\infty, w \in A_{1}$ and weak $\left(L^{1}, L^{1}\right)$ bounded.

Lemma 4 (see [12]) Let $1<p<\infty, 0<\eta<\infty, w \in A_{\infty}$ and $\Phi=\left\{\phi_{1}, \ldots, \phi_{l}\right\} \subset L^{\infty}\left(R^{n}\right)$ such that $\left|\operatorname{det}\left[\phi_{j}\left(y_{i}\right)\right]\right|^{2} \in R H_{\infty}\left(R^{n l}\right)$. Then, for any smooth function $f$ for which the left-hand side is finite,

$$
\int_{R^{n}} M_{\eta}(f)(x)^{p} w(x) d x \leq C \int_{R^{n}} M_{\Phi, \eta}^{\#}(f)(x)^{p} w(x) d x .
$$

Lemma 5 (see $[5,11]$ ) Let $1<p<\infty, w \in A_{1}$ and $0<D<2^{n}$. Then, for any smooth function $f$ for which the left-hand side is finite,

$$
\|M(f)\|_{L^{p, \varphi}(w)} \leq C\|f\|_{L^{p, \varphi}(w)} .
$$

Lemma 6 Let $1<p<\infty, 0<\eta<\infty, w \in A_{1}, 0<D<2^{n}$ and $\Phi=\left\{\phi_{1}, \ldots, \phi_{l}\right\} \subset L^{\infty}\left(R^{n}\right)$ such that $\left|\operatorname{det}\left[\phi_{j}\left(y_{i}\right)\right]\right|^{2} \in R H_{\infty}\left(R^{n l}\right)$. Then, for any smooth function $f$ for which the left-hand side is finite,

$$
\left\|M_{\eta}(f)\right\|_{L^{p, \varphi}(w)} \leq C\left\|M_{\Phi, \eta}^{\#}(f)\right\|_{L^{p, \varphi}(w)} .
$$


Proof For any cube $Q=Q\left(x_{0}, d\right)$ in $R^{n}$, we know $M\left(w \chi_{Q}\right) \in A_{1}$ for any cube $Q=Q(x, d)$ by [3]. By Lemma 4 , we have, for $f \in L^{p, \varphi}\left(R^{n}, w\right)$,

$$
\begin{aligned}
\int_{Q}\left|M_{\eta}(f)(y)\right|^{p} w(y) d y & \\
& =\int_{R^{n}}\left|M_{\eta}(f)(y)\right|^{p} w(y) \chi_{Q}(y) d y \\
& \leq \int_{R^{n}}\left|M_{\eta}(f)(y)\right|^{p} M\left(w \chi_{Q}\right)(y) d y \\
& \leq C \int_{R^{n}}\left|M_{\Phi, \eta}^{\#}(f)(y)\right|^{p} M\left(w \chi_{Q}\right)(y) d y \\
& =C\left(\int_{Q}\left|M_{\Phi, \eta}^{\#}(f)(y)\right|^{p} M\left(w \chi_{Q}\right)(y) d y+\sum_{k=0}^{\infty} \int_{2^{k+1} Q \backslash 2^{k} Q}\left|M_{\Phi, \eta}^{\#}(f)(y)\right|^{p} M\left(w \chi_{Q}\right)(y) d y\right) \\
& \leq C\left(\int_{Q}\left|M_{\Phi, \eta}^{\#}(f)(y)\right|^{p} w(y) d y+\sum_{k=0}^{\infty} \int_{2^{k+1} Q \backslash 2^{k} Q}\left|M_{\Phi, \eta}^{\#}(f)(y)\right|^{p} \frac{w(Q)}{\left|2^{k+1} Q\right|} d y\right) \\
& \leq C\left(\int_{Q}\left|M_{\Phi, \eta}^{\#}(f)(y)\right|^{p} w(y) d y+\sum_{k=0}^{\infty} \int_{2^{k+1} Q}\left|M_{\Phi, \eta}^{\#}(f)(y)\right|^{p} \frac{M(w)(y)}{2^{n(k+1)}} d y\right) \\
& \leq C\left(\int_{Q}\left|M_{\Phi, \eta}^{\#}(f)(y)\right|^{p} w(y) d y+\sum_{k=0}^{\infty} \int_{2^{k+1} Q}\left|M_{\Phi, \eta}^{\#}(f)(y)\right|^{p} \frac{w(y)}{2^{n k}} d y\right) \\
& \leq C\left\|M_{\Phi, \eta}^{\#}(f)\right\|_{L^{p, \varphi}(w)}^{p} \sum_{k=0}^{\infty} 2^{-n k} \varphi\left(2^{k+1} d\right) \\
& \leq C\left\|M_{\Phi, \eta}^{\#}(f)\right\|_{L^{p, \varphi}(w)}^{p} \sum_{k=0}^{\infty}\left(2^{-n} D\right)^{k} \varphi(d) \\
& \leq C M_{\Phi, \eta}^{\#}(f) \|_{L^{p, \varphi}(w)}^{p} \varphi(d),
\end{aligned}
$$

thus

$$
\left(\frac{1}{\varphi(d)} \int_{Q} M_{\eta}(f)(x)^{p} w(x) d x\right)^{1 / p} \leq C\left(\frac{1}{\varphi(d)} \int_{Q} M_{\Phi, \eta}^{\#}(f)(x)^{p} w(x) d x\right)^{1 / p}
$$

and

$$
\left\|M_{\eta}(f)\right\|_{L^{p, \varphi}(w)} \leq C\left\|M_{\Phi, \eta}^{\#}(f)\right\|_{L^{p, \varphi}(w)} .
$$

This finishes the proof.

Lemma 7 Let $T$ be the singular integral operator as Definition 3 or the bounded linear operator on $L^{r}(w)$ for any $1<r<\infty$ and $w \in A_{1}, 1<p<\infty, w \in A_{1}$ and $0<D<2^{n}$. Then

$$
\|T(f)\|_{L^{p, \varphi}(w)} \leq C\|f\|_{L^{p, \varphi}(w)}
$$

The proof of the lemma is similar to that of Lemma 6 by Lemma 3, we omit the details. 


\section{Proofs of theorems}

Proof of Theorem 1 It suffices to prove that for $f \in C_{0}^{\infty}\left(R^{n}\right)$ and some constant $C_{0}$, the following inequality holds:

$$
\left(\frac{1}{|Q|} \int_{Q}\left|T^{b}(f)(x)-C_{0}\right|^{r} d x\right)^{1 / r} \leq C\|b\|_{B M O} \sum_{j=1}^{m} M^{2}\left(T^{j, 2}(f)\right)(\tilde{x}),
$$

where $Q$ is any cube centered at $x_{0}, C_{0}=\sum_{j=1}^{m} \sum_{i=1}^{l} g_{j}^{i} \phi_{i}\left(x_{0}-x\right)$ and $g_{j}^{i}=\int_{R^{n}} B_{i}\left(x_{0}-\right.$ $y) M_{\left(b-b_{Q}\right) x_{(2 Q)}} T^{j, 2}(f)(y) d y$. Without loss of generality, we may assume $T^{j, 1}$ are $T(j=$ $1, \ldots, m)$. Let $\tilde{x} \in Q$. Fix a cube $Q=Q\left(x_{0}, d\right)$ and $\tilde{x} \in Q$. Write

$$
T^{b}(f)(x)=T^{b-b_{2 Q}}(f)(x)=T^{\left(b-b_{2 Q}\right) \chi_{2 Q}}(f)(x)+T^{\left(b-b_{2 Q}\right) \chi_{(2 Q)}}(f)(x)=f_{1}(x)+f_{2}(x) .
$$

Then

$$
\begin{aligned}
\left(\frac{1}{|Q|} \int_{Q}\left|T^{b}(f)(x)-C_{0}\right|^{r} d x\right)^{1 / r} \leq & C\left(\frac{1}{|Q|} \int_{Q}\left|f_{1}(x)\right|^{r} d x\right)^{1 / r} \\
& +C\left(\frac{1}{|Q|} \int_{Q}\left|f_{2}(x)-C_{0}\right|^{r} d x\right)^{1 / r}=I+I I .
\end{aligned}
$$

For $I$, by Lemmas 1,2 and 3, we obtain

$$
\begin{aligned}
& \left(\frac{1}{|Q|} \int_{Q}\left|T^{j, 1} M_{\left(b-b_{2 Q}\right) \chi_{2 Q}} T^{j, 2}(f)(x)\right|^{r} d x\right)^{1 / r} \\
& \quad \leq|Q|^{-1} \frac{\left\|T^{j, 1} M_{\left(b-b_{2 Q}\right) \chi_{2 Q}} T^{j, 2}(f) \chi_{Q}\right\|_{L^{r}}}{|Q|^{1 / r-1}} \\
& \quad \leq C|Q|^{-1}\left\|T^{j, 1} M_{\left(b-b_{2 Q}\right) \chi_{2 Q}} T^{j, 2}(f)\right\|_{W L^{1}} \\
& \quad \leq C|Q|^{-1}\left\|M_{\left(b-b_{2 Q}\right) \chi_{2 Q}} T^{j, 2}(f)\right\|_{L^{1}} \\
& \quad \leq C|Q|^{-1} \int_{2 Q}\left|b(x)-b_{2 Q} \| T^{j, 2}(f)(x)\right| d x \\
& \quad \leq C\left\|b-b_{2 Q}\right\|_{\exp L, 2 Q}\left\|T^{j, 2}(f)\right\|_{L(\log L), 2 Q} \\
& \quad \leq C\|b\|_{B M O} M^{2}\left(T^{j, 2}(f)\right)(\tilde{x}),
\end{aligned}
$$

thus

$$
I \leq C \sum_{j=1}^{m}\left(\frac{1}{|Q|} \int_{Q}\left|T^{j, 1} M_{\left(b-b_{2 Q}\right) \chi_{2 Q}} T^{j, 2}(f)(x)\right|^{r} d x\right)^{1 / r} \leq C\|b\|_{B M O} \sum_{j=1}^{m} M^{2}\left(T^{j, 2}(f)\right)(\tilde{x}) .
$$

For $I I$, we get, for $x \in Q$,

$$
\begin{aligned}
& \left|T^{j, 1} M_{\left(b-b_{Q}\right) \chi_{(2 Q)}} T^{j, 2}(f)(x)-\sum_{i=1}^{l} g_{j}^{i} \phi_{i}\left(x_{0}-x\right)\right| \\
& \quad \leq\left|\int_{R^{n}}\left(K(x-y)-\sum_{i=1}^{l} B_{i}\left(x_{0}-y\right) \phi_{i}\left(x_{0}-x\right)\right)\left(b(y)-b_{2 Q}\right) \chi_{(2 Q)}(y) T^{j, 2}(f)(y) d y\right|
\end{aligned}
$$




$$
\begin{aligned}
& \leq \sum_{k=1}^{\infty} \int_{2^{k} d \leq\left|y-x_{0}\right|<2^{k+1} d}\left|K(x-y)-\sum_{i=1}^{l} B_{i}\left(x_{0}-y\right) \phi_{i}\left(x_{0}-x\right)\right|\left|b(y)-b_{2 Q}\right|\left|T^{j, 2}(f)(y)\right| d y \\
& \leq C \sum_{k=1}^{\infty} \int_{2^{k} d \leq\left|y-x_{0}\right|<2^{k+1} d} \frac{\left|x-x_{0}\right|^{\delta}}{\left|y-x_{0}\right|^{n+\delta}}\left|b(y)-b_{2 Q}\right|\left|T^{j, 2}(f)(y)\right| d y \\
& \leq C \sum_{k=1}^{\infty} \frac{d^{\delta}}{\left(2^{k} d\right)^{n+\delta}}\left(2^{k} d\right)^{n}\left\|b-b_{2 Q}\right\|_{\exp L, 2^{k+1} Q}\left\|T^{j, 2}(f)\right\|_{L(\log L), 2^{k+1} Q} \\
& \leq C\|b\|_{B M O} M^{2}\left(T^{j, 2}(f)\right)(\tilde{x}) \sum_{k=1}^{\infty} k 2^{-k \delta} \\
& \leq C\|b\|_{B M O} M^{2}\left(T^{j, 2}(f)\right)(\tilde{x}),
\end{aligned}
$$

thus

$$
I I \leq \frac{1}{|Q|} \int_{Q} \sum_{j=1}^{m}\left|T^{j, 1} M_{\left(b-b_{Q}\right) x_{(2 Q)}} T^{j, 2}(f)(x)-C_{0}\right| d x \leq C\|b\|_{B M O} \sum_{j=1}^{m} M^{2}\left(T^{j, 2}(f)\right)(\tilde{x}) .
$$

This completes the proof of Theorem 1 .

Proof of Theorem 2 By Theorem 1 and Lemmas 3-4, we have

$$
\begin{aligned}
\left\|T^{b}(f)\right\|_{L^{p}(w)} & \leq \| M_{r}\left(\left(T^{b}(f)\right)\left\|_{L^{p}(w)} \leq C\right\| M_{\Phi, r}^{\#}\left(T^{b}(f)\right) \|_{L^{p}(w)}\right. \\
& \leq C\|b\|_{B M O} \sum_{j=1}^{m}\left\|M^{2}\left(T^{j, 2}(f)\right)\right\|_{L^{p}(w)} \leq C\|b\|_{B M O} \sum_{j=1}^{m}\left\|T^{j, 2}(f)\right\|_{L^{p}(w)} \\
& \leq C\|b\|_{B M O}\|f\|_{L^{p}(w) .}
\end{aligned}
$$

This completes the proof.

Proof of Theorem 3 By Theorem 1 and Lemmas 5-7, we have

$$
\begin{aligned}
\left\|T^{b}(f)\right\|_{L^{p, \varphi}(w)} & \leq\left\|M_{r}\left(T^{b}(f)\right)\right\|_{L^{p, \varphi}(w)} \leq C\left\|M_{\Phi, r}^{\#}\left(T^{b}(f)\right)\right\|_{L^{p, \varphi}(w)} \\
& \leq C\|b\|_{B M O} \sum_{j=1}^{m}\left\|M^{2}\left(T^{j, 2}(f)\right)\right\|_{L^{p, \varphi}(w)} \leq C\|b\|_{B M O} \sum_{j=1}^{m}\left\|T^{j, 2}(f)\right\|_{L^{p, \varphi}(w)} \\
& \leq C\|b\|_{B M O}\|f\|_{L^{p, \varphi}(w)} .
\end{aligned}
$$

This completes the proof.

\section{Competing interests}

The author declares that they have no competing interests.

Received: 4 June 2013 Accepted: 15 November 2013 Published: 17 Dec 2013

\section{References}

1. Di Fazio, G, Ragusa, MA: Interior estimates in Morrey spaces for strong solutions to nondivergence form equations with discontinuous coefficients. J. Funct. Anal. 112, 241-256 (1993) 
2. Pérez, C, Trujillo-Gonzalez, R: Sharp weighted estimates for multilinear commutators. J. Lond. Math. Soc. 65, 672-692 (2002)

3. Coifman, R, Rochberg, R: Another characterization of BMO. Proc. Am. Math. Soc. 79, 249-254 (1980)

4. Peetre, J: On the theory of $L^{p, \lambda}$-spaces. J. Funct. Anal. 4, 71-87 (1969)

5. Chiarenza, F, Frasca, M: Morrey spaces and Hardy-Littlewood maximal function. Rend. Mat. 7, $273-279$ (1987)

6. Garcia-Cuerva, J, Rubio de Francia, JL: Weighted Norm Inequalities and Related Topics. North-Holland Math. Stud. vol. 16. North-Holland, Amsterdam (1985)

7. Grubb, DJ, Moore, CN: A variant of Hörmander's condition for singular integrals. Colloq. Math. 73, 165-172 (1997)

8. Liu, LZ: Interior estimates in Morrey spaces for solutions of elliptic equations and weighted boundedness for commutators of singular integral operators. Acta Math. Sci. 25(1), 89-94 (2005)

9. Mizuhara, T: Boundedness of some classical operators on generalized Morrey spaces. In: Harmonic Analysis (Sendai, 1990). ICM-90 Satell. Conf. Proc., pp. 183-189 (1990)

10. Coifman, RR, Rochberg, R, Weiss, G: Factorization theorems for Hardy spaces in several variables. Ann. Math. 103 611-635 (1976)

11. Di Fazio, G, Ragusa, MA: Commutators and Morrey spaces. Boll. Unione Mat. Ital., A 5(7), 323-332 (1991)

12. Stein, EM: Harmonic Analysis: Real Variable Methods, Orthogonality and Oscillatory Integrals. Princeton University Press, Princeton (1993)

13. Trujillo-Gonzalez, R: Weighted norm inequalities for singular integral operators satisfying a variant of Hörmander's condition. Comment. Math. Univ. Carol. 44, 137-152 (2003)

14. Torchinsky, A: Real Variable Methods in Harmonic Analysis. Pure and Applied Math., vol. 123. Academic Press, New York (1986)

15. Chanillo, S: A note on commutators. Indiana Univ. Math. J. 31, 7-16 (1982)

10.1186/1029-242X-2013-589

Cite this article as: Feng: $M^{2}$-Type sharp estimates and boundedness on a Morrey space for Toeplitz-type operators associated to singular integral operators satisfying a variant of Hörmander's condition. Journal of Inequalities and Applications 2013, 2013:589

\section{Submit your manuscript to a SpringerOpen ${ }^{\ominus}$ journal and benefit from:}

- Convenient online submission

- Rigorous peer review

- Immediate publication on acceptance

- Open access: articles freely available online

- High visibility within the field

- Retaining the copyright to your article 